\title{
Magnetic resonance imaging-transrectal ultrasound fusion image-guided prostate biopsy: Current status of the cancer detection and the prospects of tailor-made medicine of the prostate cancer
}

\author{
Sunao Shoji \\ Department of Urology, Tokai University Hachioji Hospital, Hachioji, Tokyo, Japan
}

Multi-parametric magnetic resonance imaging (mpMRI) has been increasingly used to diagnose clinically significant prostate cancer (csPCa) because of its growing availability and its ability to combine anatomical and functional data. Magnetic resonance imaging (MRI)-transrectal ultrasound (TRUS) fusion imaging provides MRI information with TRUS images for prostate biopsies. This technique combines the superior sensitivity of MRI for targeting suspicious lesions with the practicality and familiarity of TRUS. MRI-TRUS fusion image-guided prostate biopsy is performed with different types of image registration (rigid vs. elastic) and needle tracking methods (electromagnetic tracking vs. mechanical position encoders vs. image-based software tracking). A systematic review and meta-analysis showed that MRI-targeted biopsy detected cSPCa at a significantly higher rate than did TRUS-guided biopsy, while it detected significantly fewer cases of insignificant PCas. In addition to the high accuracy of MRI-targeted biopsy for csPCa, localization of csPCa is accurate. The ability to choose the route of biopsy (transperineal vs. transrectal) is required, depending on the patients' risk and the location and size of suspicious lesions on mpMRI. Fusion image-guided prostate biopsy has the potential to allow precise management of prostate cancer, including active surveillance, radical treatment, and focal therapy.

Keywords: Biopsy; Magnetic resonance imaging; Prostatic neoplasms; Ultrasonography

This is an Open Access article distributed under the terms of the Creative Commons Attribution Non-Commercial License (http://creativecommons.org/licenses/by-nc/4.0) which permits unrestricted non-commercial use, distribution, and reproduction in any medium, provided the original work is properly cited.

\section{INTRODUCTION}

Some prostate cancers (PCas) are visible on transrectal ultrasound (TRUS) and can be targeted by an experienced ultrasonographer or urologist [1,2], but detecting clinically significant $\mathrm{PCa}$ (csPCa; defined as at least one core with a Gleason score of $3+4$ or 6 with a maximum cancer core length longer than $4 \mathrm{~mm}$ ) [3] with TRUS is difficult.
To improve the detection rate of csPCa, accurate needle placement into the cancerous lesion is essential. Recently, multi-parametric magnetic resonance imaging (mpMRI) has been increasingly used to diagnose csPCa because of its usefulness in detection with a combination of anatomical and functional data [4,5]. In the 2014 European Association of Urology guidelines, mpMRI was regarded as a particularly accurate method for detecting anterior tumors that are

Received: 11 October, 2018 - Accepted: 12 December, 2018

Corresponding Author: Sunao Shoji

Department of Urology, Tokai University Hachioji Hospital, 1838 Ishikawa-machi, Hachioji, Tokyo 192-0032, Japan

TEL: +81-42-639-1111, FAX: +81-42-639-1112, E-mail: sunashoj@mail.goo.ne.jp

ORCID: https://orcid.org/0000-0001-6673-9316 
usually missed by systematic transrectal biopsy, and thus have the risk of a repeat (targeted) biopsy [6].

Since mpMRI has improved the detection of the csPCa in the prostate [7-9], several magnetic resonance imaging (MRI)guided biopsy methods have been used, such as MRI-TRUS fusion image-guided biopsy, in-bore MRI-targeted biopsy, and cognitive biopsy. MRI-TRUS fusion imaging provides MRI information with TRUS images, which are used in prostate biopsy. This technique combines the superior visibility of MRI for targeting suspicious lesions with TRUS for real-time guidance. In-bore MRI-targeted biopsy is a direct method using MRI [10,11]. A systematic review showed no significant differences in detection of PCa between MRI-TRUS fusion image-guided biopsy and in-bore MRI-targeted biopsy $(\mathrm{p}=0.13)$, and between MRI-TRUS fusion image-guided biopsy and cognitive biopsy $(p=0.11)$ [12]. However, in-bore MRI-targeted biopsy is not acceptable in most hospitals because of its high expense and time constraints, and the frequent need for general anesthesia. Cognitive biopsy is a target biopsy based on the examiner's suspicions of a lesion on mpMRI without any fusion device. Cognitive biopsy has improved accuracy over conventional systematic biopsy [13-16]. Haffner et al. [13] reported that the accuracy of targeted biopsy detection was better than that of systematic biopsies for significant cancer detection $(p<0.001)$. They also found that the maximal cancer core length was $5.56 \mathrm{~mm}$ for targeted biopsies and $4.70 \mathrm{~mm}$ for systematic biopsies ( $\mathrm{p}=0.002$ ). Similarly, Park et al. [14] reported that cancer detection was significantly higher in cognitive biopsies (29.5\%) than in systematic biopsies (9.8\%) $(p=0.03)$. These authors also found a significantly higher positive percent core in cognitive biopsies than in systematic biopsies $(\mathrm{p}<0.001)$. In men with previous negative biopsies, the cancer detection rate of cognitive targeted biopsies (45.5\%) was significantly higher than that of systematic biopsies
$(24.4 \%)(\mathrm{p}=0.01)$ [15]. However, human error was reported as a disadvantage of cognitive biopsy when the target is less than $10 \mathrm{~mm}$ in diameter and there is the inability to track the location of each biopsy site [17]. Specialists with experience in prostate biopsy s have performed cognitive fusion biopsies with success [13-16,18].

This review article focuses on the development of mpMRI for detecting csPCa and MRI-TRUS fusion image-guided prostate biopsy. We also review the prospects for tailored treatment enabled by development of accurate detection and localization of csPCa with MRI-TRUS fusion image-guided prostate biopsy.

\section{Detection of csPCa with mpMRI}

For detection of csPCa, mpMRI can be performed at 1.5Tesla ( $\mathrm{T}$ ) or 3.0T, using either a combination of an endorectal coil and a pelvic phased-array coil, or a pelvic phased-array coil only. The use of the endorectal coil and 3.0T imaging is optional [19,20]. Currently, mpMRI includes a combination of high-resolution T2-weighted images (T2WIs), and at least two functional MRI techniques, such as diffusion-weighted images (DWIs), the apparent diffusion coefficient (ADC), or dynamic contrast-enhanced (DCE) MRI (Fig. 1) [19]. These techniques provide better characterization than T2WIs with only one functional technique [1]. Magnetic resonance spectroscopy is considered as an optional modality and has been relegated to the research setting [19]. Within mpMRI components, the relative clinical value of its component techniques differs. T2WIs provide the best depiction of the intra-prostatic structures, such as the zones, urethra, ejaculatory ducts, and capsule [1]. DCE imaging is used for evaluating tumor vascularity [21]. In a previous report, DCE was able to detect tumor vascularity in $92.9 \%$ of patients with csPCa [22]. DWI quantifies free

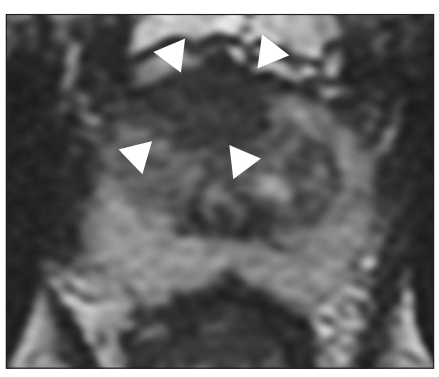

T2WI

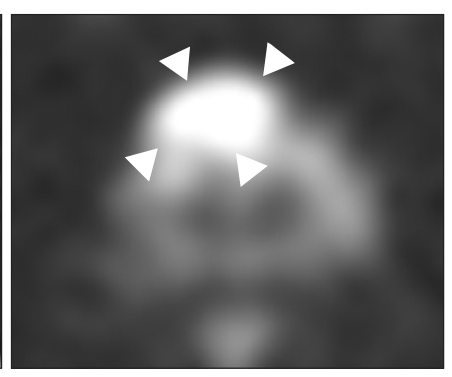

DWI

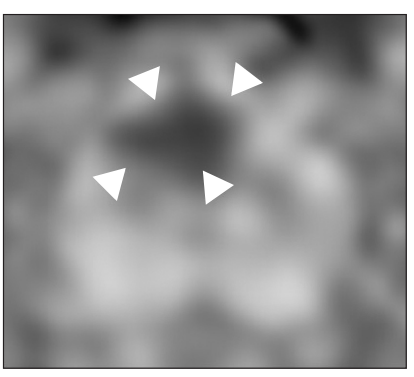

ADC

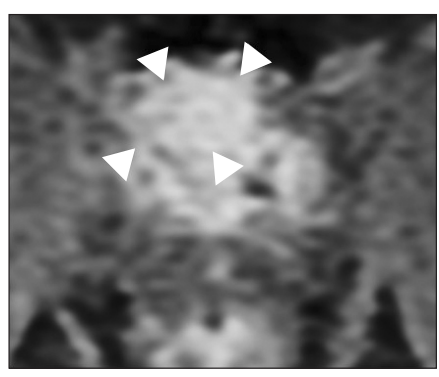

Dynamic

Fig. 1. mpMRI of a representative case suspected with prostate cancer. mpMRI includes a combination of high-resolution T2Wls, DCE images, DWls, and ADC. The lesion suspected as cancer was diagnosed in the anterior in transition zone (in the white arrows) on mpMRI, which was diagnosed as Prostate Imaging and Reporting and Data System category 5. mpMRI, multi-parametric magnetic resonance imaging; T2WI, T2-weighted image; DCE, dynamic contrast-enhanced; DWI, diffusion-weighted image; ADC, apparent diffusion coefficient. A 1.5Tesla magnet (Signa HDx) with an 8-channel cardiac coil. 
water motion, also known as 'Brownian' motion [23]. An $\mathrm{ADC}$ map is calculated from DWI, and is able to assess the aggressiveness of $\mathrm{PCa}$. The $\mathrm{ADC}$ corresponds to greater restriction in free water motion. This is likely achieved on the basis of increased cellular density compared with normal prostate tissue, and cancer tissue shows a lower ADC value than normal prostate tissue [23]. Furthermore, ADC values correlate with Gleason scores [24-26]. However, benign prostatic hyperplasia and prostatitis show high-signal intensity on DWIs and a low ADC, which suggest cancer lesions [1]. Therefore, detection of $\mathrm{PCa}$ in the transition zone is regarded as challenging [27]. Previous reports have shown that localization and pathological diagnosis of the index lesion, which is the lesion with the highest Gleason score or large volume, was accurately achieved in $>92 \%$ of patients $[28,29]$. In a comparative histological study between PCa foci that were detected and missed using mpMRI, histopathological features of cancer size, Gleason score, and solid growth were significantly different between detected and missed tumors on mpMRI $(p<0.0001)$ [30]. Independent predictors of identification of significant PCa by mpMRI were size (odds ratio [OR], 5.38; $\mathrm{p}=0.0077$ ), Gleason score $(\mathrm{OR}, 5.12 ; \mathrm{p}=0.0094)$, and solid growth $(\mathrm{OR}, 17.83$; $\mathrm{p}<0.0001)$ in multivariate analysis [30].

To standardize evaluation and reporting of mpMRI findings for diagnosing PCa, the European Society of Urogenital Radiology published guidelines, termed the Prostate Imaging and Reporting and Data System (PIRADS) [1]. PI-RADS categories range from 1 to 5 , with 5 the most likely to represent csPCa [1] In a diagnostic metaanalysis, the sensitivity and specificity were 0.78 (95\% confidence interval [C], 0.70-0.84) and 0.79 (95\% CI, 0.68-0.86), respectively [31]. A previous report showed that the areas under curves (AUCs) for cancer detection with PI-RADS in the peripheral zone and transition zone were 0.97 and 0.60 , respectively [32]. Based on these results, PI-RADS is regarded as a reliable method of diagnosing PCa. PI-RADS version 2 improves and standardizes communication between radiologists and urologists to detect or exclude the presence of csPCa with a high likelihood [33].

\section{Diagnostic accuracy of detecting csPCa with MRI-targeted biopsy}

In a systematic review, the overall PCa detection rate was not significantly different between any methods of MRI-targeted biopsy and TRUS-guided biopsy [12]. However, the detection rates of PCa per core demonstrated that MRItargeted biopsy cores had a significantly higher yield of PCa detection compared with TRUS-guided biopsy cores (relative yield: 3.91 [95\% CI, 3.17-4.83], yield of MRI-guided biopsy: 0.41 [95\% CI, 0.33-0.49], yield of TRUS-guided biopsy: 0.10 [95\% CI, 0.08-0.13]) [12]. In detection of csPCa, MRI-targeted biopsy detected csPCa significantly more frequently than did TRUSguided biopsy, with a relative sensitivity of 1.16 (95\% CI, 1.02 132; sensitivity for MRI-targeted biopsy: 0.90 [95\% CI, 0.85-0.94], sensitivity for TRUS-guided biopsy: 0.79 [95\% CI, 0.68-0.87] [12]. MRI-targeted biopsy detected significantly less insignificant PCa than did TRUS-guided biopsy, with a relative yield of 0.47 (95\% CI, 0.35-0.63; yield for MRI-guided biopsy: 0.07 [95\% CI, 0.04-0.10], yield for TRUS-guided biopsy: 0.14 [95\% CI, 0.11-0.18] [12]. With regard to comparison of MRI-targeted biopsy, in-bore MRI-targeted biopsy had a significant advantage compared with cognitive biopsy for overall detection of $\mathrm{PCa}(\mathrm{p}=0.02)$. However, there was no significant difference in detection of PCa between MRI-TRUS fusion image-guided biopsy and inbore MRI-targeted biopsy $(p=0.13)[12]$.

In a meta-analysis of 16 reports with 1,926 patients, MRI-targeted biopsy was performed with the transrectal approach based on MRI before the biopsy [34]. Additionally, TRUS-guided biopsy was performed without information of MRI, with a range of eight to 12 cores depending on prostate volume. MRI-targeted biopsy and TRUS-guided biopsy did not differ in overall PCa detection rates (sensitivity, 0.85; 95\% CI, 0.80-0.89 vs. sensitivity, 0.81; 95\% CI, 0.70-0.88) [34]. However, detection of csPCa in MRI-targeted biopsy was higher than that in TRUS-guided biopsy (sensitivity, 0.91; 95\% CI, $0.87-0.94$ vs. sensitivity, 0.76; 95\% CI, 0.64-0.84), and there was a lower rate of insignificant cancer detection (sensitivity, 0.44; 95\% CI, 0.26-0.64 vs. sensitivity, 0.83; 95\% CI, 0.77-0.87) [34]. Subgroup analysis showed an improvement in significant detection of csPCa by MRI-targeted biopsy in men with a previous negative biopsy than in men with an

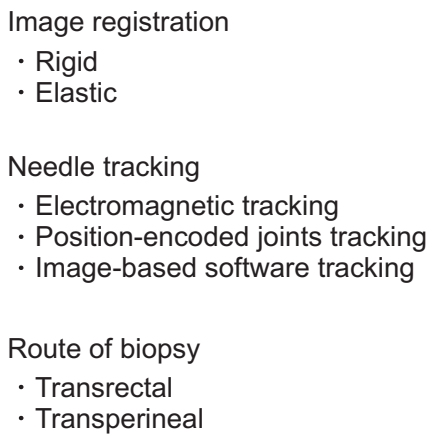

Fig. 2. Composition of MRI-TRUS fusion image-guided prostate biopsy. MRI-TRUS fusion image-guided prostate biopsy comprises image registration (rigid or elastic), the needle tracking method (electromagnetic tracking, mechanical position encoders, or image-based software tracking), and the route of biopsy (transperineal or transrectal). MRITRUS, magnetic resonance imaging-transrectal ultrasound. 
initial biopsy (relative sensitivity, 1.54; 95\% CI, 1.0-2.57 vs. relative sensitivity, 1.10; 95\% CI, 1.00-1.22) [34].

\section{Clinical results of MRI-TRUS fusion image-guid- ed prostate biopsy with different devices}

Recently, some devices of MRI-TRUS fusion imageguided prostate biopsy have become commercially available. These devices differ by type of image registration of rigid or elastic and the needle tracking method of electromagnetic tracking or mechanical position encoders vs. image-based software tracking (Fig. 2). In image registration, rigid image registration overlays mpMRI images to TRUS images during the biopsy without adjusting for deformation of the prostate owing to the pressure of the TRUS probe [35]. However, elastic fusion adopts deformation, and therefore, it is expected to be more accurate than rigid image registration (Fig. 3) [36,37]. In 2013, the BioJet (D\&K Technologies GmbH, Barum, Germany) was introduced to Japan [38].
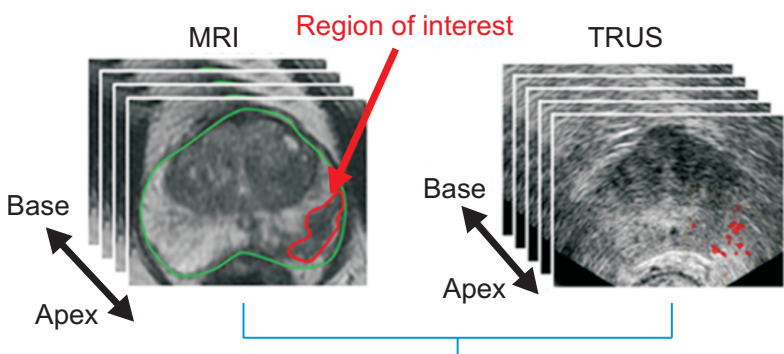

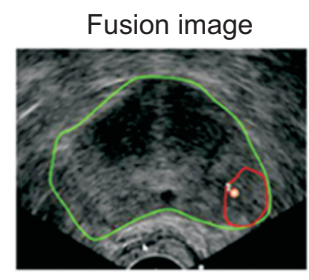

个ै

Fig. 3. Elastic fusion of MRI and TRUS. Three-dimensional information is transferred from mpMRI to TRUS of the prostate with elastic image fusion software. MRI, magnetic resonance imaging; TRUS, transrectal ultrasound; mpMRI, multi-

Elastic fusion system parametric MRI

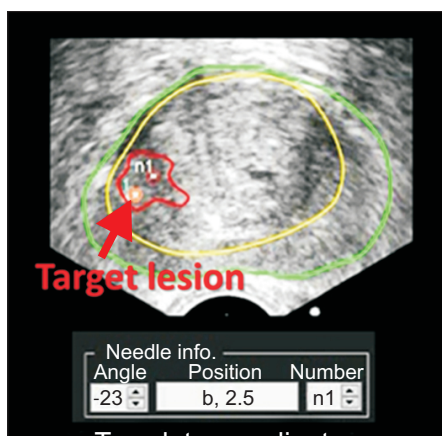

Template coordinates Navigation on axial image (2D)

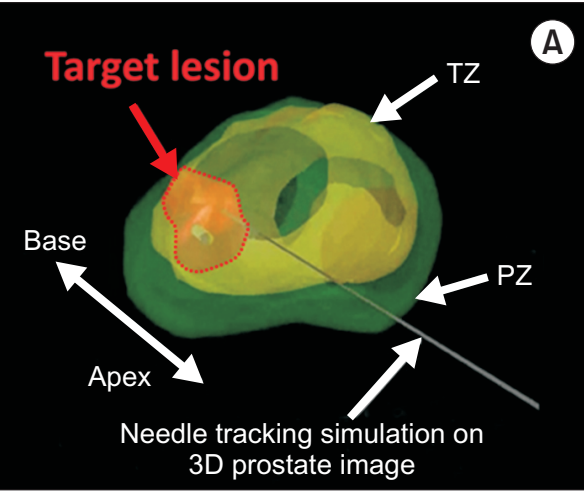

(A)

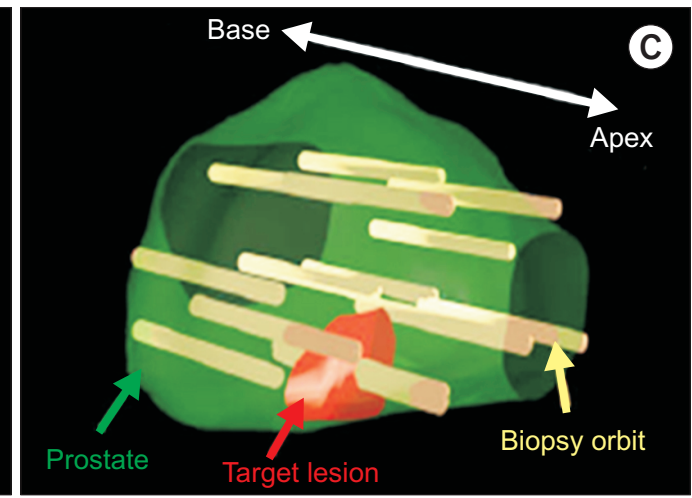

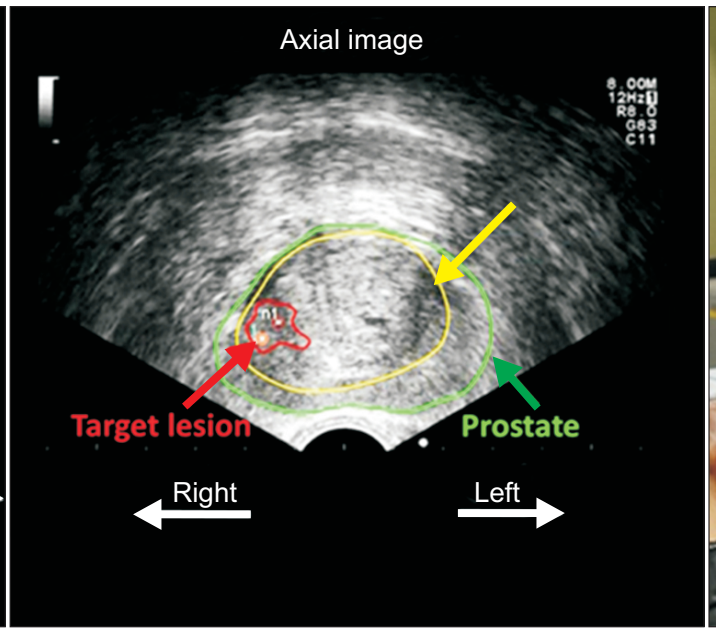

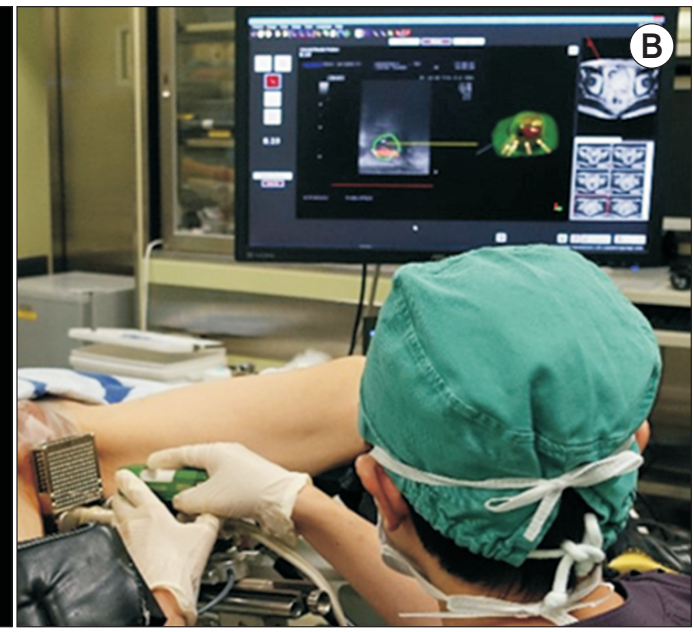

Fig. 4. Process of MRI-TRUS fusion image-guided prostate biopsy with BioJet version 2.0. (A) First, the operator marks the target point on the work station. Transperineal template coordinates are shown on the monitor. Biopsy simulation is performed in a 3D model of the prostate. (B) Transperineal template-assisted biopsy is then performed by viewing axial and sagittal real-time fusion 2D images of the prostate. For a difficult case of the template-assisted transperineal approach due to the pelvis, transperineal free-hand biopsy is performed by viewing axial and sagittal realtime fusion 2D images of the prostate. (C) After each biopsy, biopsy needle tracking is recorded in 2D and 3D fusion images of the prostate. 3D, three-dimensional; 2D, two-dimensional; TZ, transition zone; PZ, peripheral zone. 
BioJet version 2.0 induces elastic registration and uses the needle tracking method with a mechanical positionencoded stepper; elastic registration matches the corresponding point landmarks and aligns them with contours of the prostate surface [39]. Using a position-encoded mechanical stepper, the three-dimensional (3D) location of the TRUS probe is calculated by direct attachment to the mechanical stepper [39]. Angle sensors in the mechanical stepper encode and automatically relay the $3 \mathrm{D}$ position of the probe and needle to the computer [39]. Fig. 4 shows the process of an MRITRUS fusion image-guided transperineal biopsy with BioJet version 2. In our experience, the detection rate of csPCa of lesions with the PI-RADS category 5 is $80 \%$ [39]. Furthermore, detection rates for biopsy-proven csPCa among targeted lesions $\leq 10 \mathrm{~mm}$ and $>10 \mathrm{~mm}$ are similar to those with PI-RADS categories of 4 ( $50 \%$ vs. $51 \%$; $=0.707)$ and 5 ( $75 \%$ vs. $68 \%$; $p=0.386$ ) [39]. We found that receiver operating characteristic curves with PI-RADS categories showed areas under the curves (AUCs) that were significantly greater than non-discrimination for all targeted lesions (AUC, 0.835; 95\% CI, 0.792-0.879; $\mathrm{p}<0.0001$ ), lesions $\leq 10 \mathrm{~mm}$ (AUC, 0.855 ; 95\% CI, 0.805-0.905; $\mathrm{p}<0.0001$ ), and lesions $>10 \mathrm{~mm}$ (AUC, 0.835; 95\% CI, 0.844-0.903; $\mathrm{p}<0.0001$ ) [39]. Our results showed the advantages of MRI-TRUS fusion image-guided prostate biopsy. A previous report showed that, with a similar device, BiopSee (Pi Medical Ltd, Athens, Greece), transperineal MRITRUS fusion image-targeted biopsy detected 79\% of csPCas in analysis of whole-mount specimens [40]. The Artemis system (Eigen, Grass Valley, CA, USA), which uses elastic fusion and position-encoded joints in robotic arms, is used for transrectal targeted biopsy. A previous report showed that the cancer core detection rates in targeted biopsies (9.4\%) were significantly higher than those in 12 -core systematic biopsy cores (5.7\%; $p<0.05)$ [41]. Furthermore, the median lesion core length $(3.75 \mathrm{~mm}$ vs. $2.86 \mathrm{~mm} ; \mathrm{p}<0.05)$ and the percentage of cancer core length $(34.3 \%$ vs. $26.1 \%$; $p<0.05)$ in targeted biopsies were significantly higher than those in 12 core systematic biopsies [41]. The UroNav system (Invivo, Gainesville, FL, USA) uses elastic fusion and electromagnetic needle tracking. A previous study showed that transrectal target biopsies with the UroNav $(\mathrm{n}=461)$ diagnosed $30 \%$ more PCas with a Gleason score $\geq 4+3$ than did 12-core template systematic biopsies ( $\mathrm{n}=469)(173$ cases vs. 122 cases; $\mathrm{p}<0.001)$ [42]. In another report, the cancer detection rate for transrectal targeted biopsy using the UroNav system was significantly higher $(48.1 \%, 39 / 81$ patients) than that for transrectal biopsy with visual registration (34.7\%, 52/150 patients; $\mathrm{p}=0.04$ ) or 12-core transrectal systematic biopsy (32.0\%, 32/100 patients; $p=0.03$ ) [43]. In the Urostation system
(Koelis, Meylan, France), elastic fusion and 3D TRUS image needle tracking are used [44]. A previous study showed that targeted biopsies with the Urostation had significantly higher overall cancer detection rates $(31 \%$ vs. $7.5 \%$; $p<0.001)$ and detection rates for clinically significant cancers ( $43 \%$ vs. $37 \%$; $\mathrm{p}=0.03$ ) than did 12 -core systematic biopsy in 152 patients with prostate-specific antigen levels $<10 \mathrm{ng} / \mathrm{mL}$ [45]. Additionally, the cancer core length in targeted biopsy cores (median, $8 \mathrm{~mm}$ ) was significantly higher than that in systematic biopsy cores (median, $4 \mathrm{~mm}$; $\mathrm{p}<0.0001, \mathrm{n}=152$ ) [45].

In localization of csPCa in the prostate, concordance between the index lesion location on biopsy and radical prostatectomy specimens is $95 \%$. A previous study investigated 135 patients who had cancer diagnosed with T2WI and DWI-ADC sequences on $1.5 \mathrm{~T}$ or 3.0T MRI [29]. This study showed that concordance of the primary Gleason pattern between targeted biopsy and radical prostatectomy specimens was $90 \%$ based on the results of MRI-TRUS fusion image-guided biopsy.

\section{Controversy in MRI-TRUS fusion image-guided prostate biopsy}

\section{1) Approach of the biopsy: transperineal or tran- srectal?}

There are no clear recommendations regarding the transrectal or transperineal approach. For systematic TRUSguided biopsy, cancer detection rates are comparable for both approaches $[46,47]$. With regard to complications, the incidence rates of infection and rectal bleeding are higher with transrectal prostate biopsy than with the transperineal approach [48]. Furthermore, lesions that are located in the anterior part of the prostate, particularly in high-volume prostates, might be missed in transrectal prostate biopsy. Transperineal prostate biopsy usually ensures greater comfort of the patient. Previous studies have suggested that transrectal biopsy might have a higher risk of infection than the transperineal approach because fecal bacteria can enter the blood circulation after retrieving specimens from the prostate [49]. Transperineal biopsy has been reported to be more painful than transrectal biopsy [50]. Therefore, spinal anesthesia is performed for transperineal biopsy and thus it might not be suitable for outpatient procedures. However, few groups have reported the possibility of obtaining a transperineal biopsy under local anesthesia [51].

Tewes et al. [52] introduced the process of the decision for which biopsy approach to use (transperineal vs. transrectal) with BioJet. In this process, the approach was decided on the basis of the patient's wishes after detailed information of the 
risks and advantages of the transrectal and transperineal approaches [52]. Additionally, these authors considered that prostate size, tumor localization according to MRI, and the patients' general condition were important for the decision [52] They achieved a high detection rate of PCa. They found that sensitivity/specificity for tumor detection with a PIRADS category $\geq 4$ were $81 \% / 69 \%$ for transrectal biopsy and $86 \% / 84 \%$ for transperineal biopsy [52]. Based on previous studies, a flexible choice of the biopsy approach is required depending on the patients' risk (e.g, poorly controlled case of diabetes mellitus, hemorrhoids), and the location and size of suspected cancer lesions on mpMRI.

\section{2) Image registration in MRI and TRUS: elastic fu- sion vs. rigid?}

Because elastic fusion compensates for possible deformation caused by insertion of the ultrasound probe into the rectum, it is expected to perform better than rigid registration. A systematic review and meta-analysis evaluated elastic versus rigid image registration in MRITRUS fusion image-guided prostate biopsy [53]. This analysis showed that the detection odds ratios for significant $\mathrm{PCa}$ were 1.45 (95\% CI, 1.21-1.73; p<0.0001) and 1.40 (95\% CI, $1.13-1.75 ; p=0.002)$ for the elastic and the rigid registration subgroups, respectively, and there was no significant difference between the subgroups $(p=0.83)$. However, rigid registration requires cognitive optimization of registration, and accurate cognitive fusion should be performed after rigid software-assisted fusion. Therefore, rigid registration would be appropriate for experts in cognitive fusion, but not for those without this knowledge. Because there have been no prospective comparisons between elastic and rigid image registration, a randomized, prospective, comparative study is required to investigate this issue.

\section{Prospects of tailor-made medicine of PCa by development of MRI-targeted biopsy}

With development of MRI-targeted biopsy, high accuracy of detection and spatial location of csPCa has been achieved. This development has contributed to management of PCa, such as active surveillance, radical prostatectomy, and focal therapy. Active surveillance is a standard option for patients with low-risk PCa [54]. Currently, serum prostatespecific antigen kinetics [55] and pathological findings [56] of follow-up systematic biopsy are monitored in patients under active surveillance. A limitation of the present active surveillance is that prostate-specific antigen kinetics is not cancer-specific, with fluctuation by inflammation and mechanical manipulation. Additionally, systematic biopsy lacks reliability for predicting disease progression during active surveillance [56]. Therefore, image-based active surveillance has been reported. In a retrospective study of image-based monitoring of targeted biopsy-proven PCa in active surveillance, the potential of longitudinal monitoring of TRUS-visible biopsy-proven cancer was reported in an 11year experience [57]. In multivariate analysis for predicting pathological progression, an increase of $\geq 25 \%$ in diameter of the biopsy-proven lesion (hazard ratio, 15.314; $\mathrm{p}=0.023$ ) and an upgrade of Doppler grade (hazard ratio, 37.409; $\mathrm{p}=0.019$ ) were significant risk factors [57]. Clinical studies of mpMRIbased active surveillance are ongoing because mpMRI is a promising risk stratification tool and should be considered for men on active surveillance [58]. In radical prostatectomy, the usefulness of real-time monitoring of TRUS-visible csPC lesions during robot-assisted radical prostatectomy has been reported [59]. A clinical study showed that three of four patients who were highly suspected as having capsule invasion achieved a negative surgical margin [59]. This was shown by real-time navigation of the TRUS-visible csPC

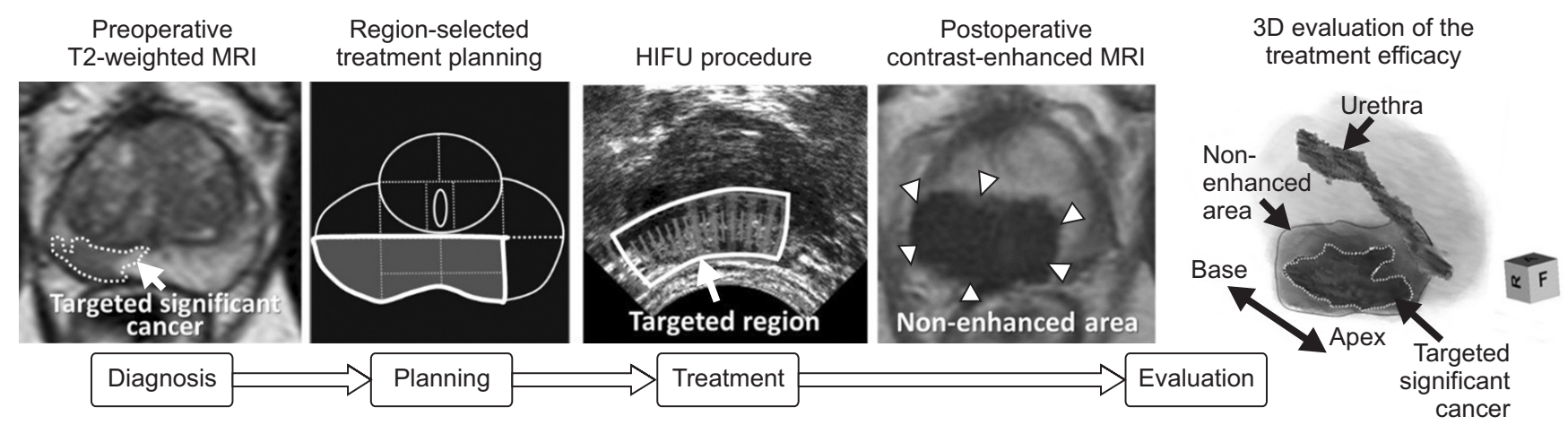

Fig. 5. Process of focal therapy for localized prostate cancer with high-intensity focused ultrasound. MRI-TRUS elastic fusion image-guided transperineal biopsy is performed with the BioJet system. The recorded localization of mpMRI-visible csPCa is converted to the treatment planning screen of TRUS images on a workstation of the HIFU device (Sonablate 500). The treatment range is determined and csPCa is treated. Contrastenhanced MRI is performed at 2 weeks after treatment for evaluation of the treatment effect. MRI-TRUS, magnetic resonance imaging-transrectal ultrasound; mpMRI, multi-parametric magnetic resonance imaging; 3D, three-dimensional. 
location during robot-assisted radical prostatectomy.

Multi-focality of PCa has been reported to be as high as $80 \%$ [60]. However, recent evidence suggests that csPCa is the responsible lesion for disease progression [61,62]. Therefore, treatment of csPCa would potentially achieve control of tumor progression. Based on the concept and development of diagnosis of csPCa, clinical trials of focal therapy have been conducted for localized PCa treatment [63]. The aim of focal therapy is to treat clinical significant cancer with minimal injury to the urethra, sphincter, neurovascular bundle, and bladder neck [64]. Although there have been a few clinical studies in Asia, only one prospective clinical trial of focal therapy with high-intensity focused ultrasound (HIFU) based on MRI-TRUS elastic fusion image-guided transperineal prostate biopsy has been reported [65]. Fig. 5 shows the treatment process of focal therapy for localized PCa with HIFU by a Japanese group.

\section{CONCLUSIONS}

MRI-TRUS fusion image-guided prostate biopsy achieves higher accuracy of detection and spatial location of csPCa compared with systematic biopsy. With development of biopsies, prostate management could progress to precise tailor-made medicine, such as active surveillance, radical treatment, and focal therapy.

\section{CONFLICTS OF INTEREST}

The author has nothing to disclose.

\section{ACKNOWLEDGMENTS}

This work was supported by the Takeda Science Foundation.

We thank Ellen Knapp, PhD, from Edanz Group (www. edanzediting.com/ac) for editing a draft of this manuscript.

\section{REFERENCES}

1. Barentsz JO, Richenberg J, Clements R, Choyke P, Verma S, Villeirs G, et al. ESUR prostate MR guidelines 2012. Eur Radiol 2012;22:746-57.

2. Toi A, Neill MG, Lockwood GA, Sweet JM, Tammsalu LA, Fleshner NE. The continuing importance of transrectal ultrasound identification of prostatic lesions. J Urol 2007;177:51620.

3. Dickinson L, Ahmed HU, Allen C, Barentsz JO, Carey B, Futterer JJ, et al. Magnetic resonance imaging for the detection, localisation, and characterisation of prostate cancer: recommendations from a European consensus meeting. Eur Urol 2011;59:477-94.

4. Vilanova JC, Barceló-Vidal C, Comet J, Boada M, Barceló J, Ferrer J, et al. Usefulness of prebiopsy multifunctional and morphologic MRI combined with free-to-total prostate-specific antigen ratio in the detection of prostate cancer. AJR Am J Roentgenol 2011;196:W715-22.

5. Delongchamps NB, Rouanne M, Flam T, Beuvon F, Liberatore $\mathrm{M}$, Zerbib $\mathrm{M}$, et al. Multiparametric magnetic resonance imaging for the detection and localization of prostate cancer: combination of T2-weighted, dynamic contrast-enhanced and diffusion-weighted imaging. BJU Int 2011;107:1411-8.

6. Mottet N, Clarke N, De Santis M, Zattoni F, Morote J, Joniau S. Implementing newer agents for the management of castrateresistant prostate cancer: what is known and what is needed? BJU Int 2015;115:364-72.

7. Moore CM, Kasivisvanathan V, Eggener S, Emberton M, Fütterer JJ, Gill IS, et al. Standards of reporting for MRI-targeted biopsy studies (START) of the prostate: recommendations from an International Working Group. Eur Urol 2013;64:54452.

8. Sciarra A, Barentsz J, Bjartell A, Eastham J, Hricak H, Panebianco $\mathrm{V}$, et al. Advances in magnetic resonance imaging: how they are changing the management of prostate cancer. Eur Urol 2011;59:962-77.

9. Hambrock T, Somford DM, Huisman HJ, van Oort IM, Witjes JA, Hulsbergen-van de Kaa CA, et al. Relationship between apparent diffusion coefficients at 3.0-T MR imaging and Gleason grade in peripheral zone prostate cancer. Radiology 2011;259:453-61.

10. Kasivisvanathan V, Dufour R, Moore CM, Ahmed HU, AbdAlazeez M, Charman SC, et al. Transperineal magnetic resonance image targeted prostate biopsy versus transperineal template prostate biopsy in the detection of clinically significant prostate cancer. J Urol 2013;189:860-6.

11. Hambrock T, Somford DM, Hoeks C, Bouwense SA, Huisman $\mathrm{H}$, Yakar D, et al. Magnetic resonance imaging guided prostate biopsy in men with repeat negative biopsies and increased prostate specific antigen. J Urol 2010;183:520-7.

12. Wegelin O, van Melick HHE, Hooft L, Bosch JLHR, Reitsma HB5, Barentsz JO, et al. Comparing three different techniques for magnetic resonance imaging-targeted prostate biopsies: a systematic review of in-bore versus magnetic resonance imaging-transrectal ultrasound fusion versus cognitive registration. Is there a preferred technique? Eur Urol 2017;71:517-31.

13. Haffner J, Lemaitre L, Puech P, Haber GP, Leroy X, Jones JS, et al. Role of magnetic resonance imaging before initial biopsy: comparison of magnetic resonance imaging-targeted and sys- 
tematic biopsy for significant prostate cancer detection. BJU Int 2011;108:E171-8.

14. Park BK, Park JW, Park SY, Kim CK, Lee HM, Jeon SS, et al. Prospective evaluation of 3-T MRI performed before initial transrectal ultrasound-guided prostate biopsy in patients with high prostate-specific antigen and no previous biopsy. AJR Am J Roentgenol 2011;197:W876-81.

15. Sciarra A, Panebianco V, Ciccariello M, Salciccia S, Cattarino $\mathrm{S}$, Lisi D, et al. Value of magnetic resonance spectroscopy imaging and dynamic contrast-enhanced imaging for detecting prostate cancer foci in men with prior negative biopsy. Clin Cancer Res 2010;16:1875-83.

16. Labanaris AP, Engelhard K, Zugor V, Nützel R, Kühn R. Prostate cancer detection using an extended prostate biopsy schema in combination with additional targeted cores from suspicious images in conventional and functional endorectal magnetic resonance imaging of the prostate. Prostate Cancer Prostatic Dis 2010;13:65-70.

17. Sonn GA, Margolis DJ, Marks LS. Target detection: magnetic resonance imaging-ultrasound fusion-guided prostate biopsy. Urol Oncol 2014;32:903-11.

18. Moore CM, Robertson NL, Arsanious N, Middleton T, Villers A, Klotz L, et al. Image-guided prostate biopsy using magnetic resonance imaging-derived targets: a systematic review. Eur Urol 2013;63:125-40.

19. Weinreb JC, Barentsz JO, Choyke PL, Cornud F, Haider MA, Macura KJ, et al. PI-RADS prostate imaging - reporting and data system: 2015, version 2. Eur Urol 2016;69:16-40.

20. Barret E, Turkbey B, Puech P, Durand M, Panebianco V, Fütterer JJ, et al. Update on the ICUD-SIU consultation on multi-parametric magnetic resonance imaging in localised prostate cancer. World J Urol 2018 Jul 12 [Epub]. http://doi. org/10.1007/S00345-018-2395-3.

21. Collins DJ, Padhani AR. Dynamic magnetic resonance imaging of tumor perfusion. Approaches and biomedical challenges. IEEE Eng Med Biol Mag 2004;23:65-83.

22. Hara N, Okuizumi M, Koike H, Kawaguchi M, Bilim V. Dynamic contrast-enhanced magnetic resonance imaging (DCEMRI) is a useful modality for the precise detection and staging of early prostate cancer. Prostate 2005;62:140-7.

23. Gibbs P, Liney GP, Pickles MD, Zelhof B, Rodrigues G, Turnbull LW. Correlation of ADC and T2 measurements with cell density in prostate cancer at 3.0 Tesla. Invest Radiol 2009;44:572-6.

24. van As NJ, de Souza NM, Riches SF, Morgan VA, Sohaib SA, Dearnaley DP, et al. A study of diffusion-weighted magnetic resonance imaging in men with untreated localised prostate cancer on active surveillance. Eur Urol 2009;56:981-7.

25. Zelhof B, Pickles M, Liney G, Gibbs P, Rodrigues G, Kraus S, et al. Correlation of diffusion-weighted magnetic resonance data with cellularity in prostate cancer. BJU Int 2009;103:883-8.

26. Turkbey B, Shah VP, Pang Y, Bernardo M, Xu S, Kruecker J, et al. Is apparent diffusion coefficient associated with clinical risk scores for prostate cancers that are visible on 3-T MR images? Radiology 2011;258:488-95.

27. Oto A, Kayhan A, Jiang Y, Tretiakova M, Yang C, Antic T, et al. Prostate cancer: differentiation of central gland cancer from benign prostatic hyperplasia by using diffusion-weighted and dynamic contrast-enhanced MR imaging. Radiology 2010;257: 715-23.

28. Rud E, Klotz D, Rennesund K, Baco E, Berge V, Lien D, et al. Detection of the index tumour and tumour volume in prostate cancer using T2-weighted and diffusion-weighted magnetic resonance imaging (MRI) alone. BJU Int 2014;114:E32-42.

29. Baco E, Ukimura O, Rud E, Vlatkovic L, Svindland A, Aron $\mathrm{M}$, et al. Magnetic resonance imaging-transectal ultrasound image-fusion biopsies accurately characterize the index tumor: correlation with step-sectioned radical prostatectomy specimens in 135 patients. Eur Urol 2015;67:787-94.

30. Rosenkrantz AB, Mendrinos S, Babb JS, Taneja SS. Prostate cancer foci detected on multiparametric magnetic resonance imaging are histologically distinct from those not detected. J Urol 2012;187:2032-8.

31. Hamoen EH, de Rooij M, Witjes JA, Barentsz JO, Rovers MM. Use of the Prostate Imaging Reporting and Data System (PIRADS) for prostate cancer detection with multiparametric magnetic resonance imaging: a diagnostic meta-analysis. Eur Urol 2015;67:1112-21.

32. Junker D, Quentin M, Nagele U, Edlinger M, Richenberg J, Schaefer G, et al. Evaluation of the PI-RADS scoring system for mpMRI of the prostate: a whole-mount step-section analysis. World J Urol 2015;33:1023-30.

33. Steiger P, Thoeny HC. Prostate MRI based on PI-RADS version 2: how we review and report. Cancer Imaging 2016;16:9.

34. Schoots IG, Roobol MJ, Nieboer D, Bangma CH, Steyerberg EW, Hunink MG. Magnetic resonance imaging-targeted biopsy may enhance the diagnostic accuracy of significant prostate cancer detection compared to standard transrectal ultrasoundguided biopsy: a systematic review and meta-analysis. Eur Urol 2015;68:438-50.

35. Delongchamps NB, Peyromaure M, Schull A, Beuvon F, Bouazza N, Flam T, et al. Prebiopsy magnetic resonance imaging and prostate cancer detection: comparison of random and targeted biopsies. J Urol 2013;189:493-9.

36. Rud E, Baco E, Eggesbø HB. MRI and ultrasound-guided prostate biopsy using soft image fusion. Anticancer Res 2012;32:3383-9.

37. Logan JK, Rais-Bahrami S, Turkbey B, Gomella A, Amalou H, 
Choyke PL, et al. Current status of magnetic resonance imaging (MRI) and ultrasonography fusion software platforms for guidance of prostate biopsies. BJU Int 2014;114:641-52.

38. Shoji S, Hiraiwa S, Endo J, Hashida K, Tomonaga T, Nakano M, et al. Manually controlled targeted prostate biopsy with realtime fusion imaging of multiparametric magnetic resonance imaging and transrectal ultrasound: an early experience. Int J Urol 2015;22:173-8.

39. Shoji S, Hiraiwa S, Ogawa T, Kawakami M, Nakano M, Hashida K, et al. Accuracy of real-time magnetic resonance imagingtransrectal ultrasound fusion image-guided transperineal target biopsy with needle tracking with a mechanical positionencoded stepper in detecting significant prostate cancer in biopsy-naïve men. Int J Urol 2017;24:288-94.

40. Radtke JP, Schwab C, Wolf MB, Freitag MT, Alt CD, Kesch C, et al. Multiparametric magnetic resonance imaging (MRI) and MRI-transrectal ultrasound fusion biopsy for index tumor detection: correlation with radical prostatectomy specimen. Eur Urol 2016;70:846-53.

41. Wysock JS, Rosenkrantz AB, Huang WC, Stifelman MD, Lepor $\mathrm{H}$, Deng FM, et al. A prospective, blinded comparison of magnetic resonance (MR) imaging-ultrasound fusion and visual estimation in the performance of MR-targeted prostate biopsy: the PROFUS trial. Eur Urol 2014;66:343-51.

42. Siddiqui MM, Rais-Bahrami S, Turkbey B, George AK, Rothwax J, Shakir N, et al. Comparison of MR/ultrasound fusionguided biopsy with ultrasound-guided biopsy for the diagnosis of prostate cancer. JAMA 2015;313:390-7.

43. Oberlin DT, Casalino DD, Miller FH, Matulewicz RS, Perry KT, Nadler RB, et al. Diagnostic value of guided biopsies: fusion and cognitive-registration magnetic resonance imaging versus conventional ultrasound biopsy of the prostate. Urology 2016;92:75-9.

44. Kongnyuy M, George AK, Rastinehad AR, Pinto PA. Magnetic resonance imaging-ultrasound fusion-guided prostate biopsy: review of technology, techniques, and outcomes. Curr Urol Rep 2016;17:32.

45. Mozer P, Rouprêt M, Le Cossec C, Granger B, Comperat E, de Gorski A, et al. First round of targeted biopsies using magnetic resonance imaging/ultrasonography fusion compared with conventional transrectal ultrasonography-guided biopsies for the diagnosis of localised prostate cancer. BJU Int 2015;115:507.

46. Hara R, Jo Y, Fujii T, Kondo N, Yokoyoma T, Miyaji Y, et al. Optimal approach for prostate cancer detection as initial biopsy: prospective randomized study comparing transperineal versus transrectal systematic 12-core biopsy. Urology 2008;71:191-5.

47. Takenaka A, Hara R, Ishimura T, Fujii T, Jo Y, Nagai A, et al.
A prospective randomized comparison of diagnostic efficacy between transperineal and transrectal 12-core prostate biopsy. Prostate Cancer Prostatic Dis 2008;11:134-8.

48. Huang H, Wang W, Lin T, Zhang Q, Zhao X, Lian H, et al. Comparison of the complications of traditional 12 cores transrectal prostate biopsy with image fusion guided transperineal prostate biopsy. BMC Urol 2016;16:68.

49. Steensels D, Slabbaert K, De Wever L, Vermeersch P, Van Poppel H, Verhaegen J. Fluoroquinolone-resistant E. coli in intestinal flora of patients undergoing transrectal ultrasound-guided prostate biopsy-should we reassess our practices for antibiotic prophylaxis? Clin Microbiol Infect 2012;18:575-81.

50. Guo LH, Wu R, Xu HX, Xu JM, Wu J, Wang S, et al. Comparison between ultrasound guided transperineal and transrectal prostate biopsy: a prospective, randomized, and controlled trial. Sci Rep 2015 Nov 3 [Epub]. http://doi.org/10.1038/srep16089.

51. Smith JB, Popert R, Nuttall MC, Vyas L, Kinsella J, Cahill D. Transperineal sector prostate biopsies: a local anesthetic outpatient technique. Urology 2014;83:1344-9.

52. Tewes S, Peters I, Tiemeyer A, Peperhove M1, Hartung D1, Pertschy S, et al. Evaluation of MRI/ultrasound fusion-guided prostate biopsy using transrectal and transperineal approaches. Biomed Res Int 2017;2017:2176471.

53. Venderink W, de Rooij M, Sedelaar JPM, Huisman HJ, Fütterer JJ. Elastic versus rigid image registration in magnetic resonance imaging-transrectal ultrasound fusion prostate biopsy: a systematic review and meta-analysis. Eur Urol Focus 2018;4:21927.

54. Mohler JL; NCCN Prostate Cancer Panel. Joint statement by members of the NCCN Prostate Cancer Guidelines Panel. J Natl Compr Canc Netw 2013;11:1310-2.

55. van den Bergh RC, Roemeling S, Roobol MJ, Aus G, Hugosson J, Rannikko AS, et al. Outcomes of men with screen-detected prostate cancer eligible for active surveillance who were managed expectantly. Eur Urol 2009;55:1-8.

56. Porten SP, Whitson JM, Cowan JE, Perez N, Shinohara K, Carroll PR. Changes in cancer volume in serial biopsies of men on active surveillance for early stage prostate cancer. J Urol 2011;186:1825-9.

57. Shoji S, Ukimura O, de Castro Abreu AL, Marien A, Matsugasumi T, Bahn D, et al. Image-based monitoring of targeted biopsy-proven prostate cancer on active surveillance: 11-year experience. World J Urol 2016;34:221-7.

58. Stavrinides V, Giganti F, Emberton M, Moore CM. MRI in active surveillance: a critical review. Prostate Cancer Prostatic Dis 2018 Aug 16 [Epub]. http://doi.org/10.1038/s41391-0180077-2.

59. Hung AJ, Abreu AL, Shoji S, Goh AC, Berger AK, Desai MM, et al. Robotic transrectal ultrasonography during robot-assist- 
ed radical prostatectomy. Eur Urol 2012;62:341-8.

60. Humphrey PA. Complete histologic serial sectioning of a prostate gland with adenocarcinoma. Am J Surg Pathol 1993;17:468-72.

61. Gangi A, Tsoumakidou G, Abdelli O, Buy X, de Mathelin M, Jacqmin D, et al. Percutaneous MR-guided cryoablation of prostate cancer: initial experience. Eur Radiol 2012;22:182935.

62. Ahmed HU, Hindley RG, Dickinson L, Freeman A, Kirkham AP, Sahu M, et al. Focal therapy for localised unifocal and multifocal prostate cancer: a prospective development study. Lancet Oncol 2012;13:622-32.
63. Muto S, Yoshii T, Saito K, Kamiyama Y, Ide H, Horie S. Focal therapy with high-intensity-focused ultrasound in the treatment of localized prostate cancer. Jpn J Clin Oncol 2008;38:192-9.

64. Ahmed HU, Freeman A, Kirkham A, Sahu M, Scott R, Allen C, et al. Focal therapy for localized prostate cancer: a phase I/II trial. J Urol 2011;185:1246-54.

65. Shoji S, Mouraviev V, Scionti S. High intensity focused ultrasound (HIFU) treatment of prostate cancer. In: Johansen TEB, Greene D, Breen DJ, Mouraviev V. Handbook of Focal Therapy for Prostate and Renal Cancer. London: JP Medical Ltd, 2016;241-54. 